\title{
Escalas de Medida da Percepção da Qualidade do Ambiente Hospitalar - Um Estudo em Unidades de Dor
}

\author{
Rita Morais ${ }^{1}$ \\ Cláudia Campos Andrade \\ Sónia Bernardes \\ Instituto Universitário de Lisboa \\ Cícero Roberto Pereira \\ Universidade Federal da Paraíba
}

\begin{abstract}
RESUMO - Este estudo desenvolve e valida duas escalas de medida da percepção da qualidade do ambiente hospitalar: físico e sócio-funcional. Participaram 122 utilizadores de Unidades de Dor Portuguesas. Os resultados de Análises Fatoriais Confirmatórias atestam a validade fatorial da escala de percepção de qualidade do ambiente físico composta por cinco fatores: Conforto físico-espacial, Orientação, Tranquilidade, Vista e iluminação e Temperatura e qualidade do ar; e da escala de percepção de qualidade do ambiente sócio-funcional composta por dois fatores: Relações sociais e organizacionais e Privacidade. Sendo a qualidade do ambiente hospitalar um importante fator para o bem-estar, a demonstração da validade fatorial dessas escalas é útil para o estudo e promoção da qualidade de ambientes hospitalares saudáveis.
\end{abstract}

Palavras-chave: escala, hospital, ambiente físico, ambiente sócio-funcional

\section{Scales of Perceived Quality of Hospital Environment - A Study in Pain Care Units}

\begin{abstract}
This study aimed to improve and validate two scales measuring perceived quality of hospitals' physical and sociofunctional environments. Two hundred and twenty-two users of Portuguese Pain Care Units participated in the study. The results obtained by Confirmatory Factor Analysis confirmed the factor structure of the perceived quality of the physical environment scale, composed by five factors: Spatial-physical comfort, Orientation, Quietness, Views and lighting and Temperature and air quality; and the factor structure of the perceived quality of social-functional environment scale, composed by two factors: Social and Organizational Relationships and Privacy. Because the quality of hospital environment is an important predictor of users' well-being, the factorial validity of these scales is useful for the study and promotion of healthier hospital environments.
\end{abstract}

Keywords: scale, hospital, physical environment, socio-functional environment

A investigação em Psicologia Ambiental tem mostrado que as características do ambiente físico hospitalar podem influenciar de forma significativa a saúde física e psicológica dos seus utilizadores (para uma revisão, ver Devlin \& Arneill, 2003). Vários estudos revelam que um ambiente físico hospitalar adequado assegura não apenas condições de bem-estar facilitadoras da recuperação dos doentes (e.g., Ulrich, 1984), como melhora as avaliações que estes fazem acerca dos profissionais de saúde, aumenta a sua satisfação com o serviço e a percepção da qualidade dos cuidados (e.g., Andrade, Lima, Pereira, Fornara, \& Bonaiuto, 2013). Em relação aos profissionais de saúde, o seu desempenho (e.g., Chaudhury, Mahmood, \& Valente, 2009) e a sua satisfação com o trabalho (e.g., Andrade, Hernandez-Fernaud, \& Lima, 2013) dependem em parte da qualidade do ambiente físico hospitalar. Assim, é relevante compreender, avaliar e monitorizar as percepções dos doentes e dos profissionais de saúde acerca da qualidade do ambiente físico hospitalar e para isso são necessários instrumentos de medida válidos e fidedignos. Tais instrumentos possibilitam o estudo do papel desempenhado pela qualidade subjetiva do ambiente

1 Endereço para correspondência: Centro de Investigação e Intervenção Social, Instituto Universitário de Lisboa, Edifício ISCTE, Av. das Forças Armadas, Lisboa, Portugal. CEP: 1649-026. E-mail: rita_ margarida_morais@iscte.pt hospitalar na satisfação, saúde e bem-estar dos utilizadores, bem como permite avaliar intervenções ambientais centradas em criar ambientes de cuidado que vão ao encontro das necessidades e preferências dos doentes e dos profissionais de saúde. Seguindo essa orientação, o estudo que aqui se apresenta teve como objetivo dar continuidade ao estudo e desenvolvimento do instrumento Perceived Hospital Environment Quality Indicators (PHEQI) com base numa amostra de utilizadores de Unidades de Dor.

\section{O Desenvolvimento e Validação do PHEQI}

A Percepção da Qualidade Ambiental (PQA) é definida como a avaliação que os utilizadores fazem de determinado ambiente físico tendo em conta a sua experiência e considerando em que medida esse ambiente corresponde às suas necessidades e objetivos (e.g., Bell, Greene, Fisher, \& Baum, 2001; Bonnes \& Secchiaroli, 1995). A PQA é geralmente obtida através de medidas de auto-relato em que se solicita a avaliação subjetiva de vários atributos específicos do ambiente físico e social. Ao longo do tempo têm sido desenvolvidas diversas escalas para avaliar a qualidade de diferentes tipos de ambientes, como por exemplo, ambientes residenciais (e.g., Bonaiuto, Fornara, \& Bonnes, 2003) e ambientes institucionais (e.g., Moos \& Lemke, 1984). 
O estudo da PQA de ambientes hospitalares é mais recente e traduziu-se no surgimento do PHEQI (Fornara, Bonaiuto, \& Bonnes, 2006). Esse instrumento foi desenvolvido em Itália com uma amostra de utilizadores de três serviços de Ortopedia. O instrumento original é composto por quatro escalas, três focadas em aspectos do ambiente físico e uma focada no ambiente social: a) Aspectos físico-espaciais do espaço exterior do hospital (16 itens); b) Aspectos físicoespaciais do serviço em geral (21 itens); c) Aspectos físicoespaciais da área de internamento/ área de espera do serviço de consulta (18 itens) e d) Aspectos sócio-funcionais (18 itens). Recorrendo a uma análise em componentes principais, Fornara et al. (2006) identificaram 12 fatores de PQA, nomeadamente: Manutenção e cuidado, Orientação, Estética do edifício e Espaços verdes, em relação ao espaço exterior do hospital (15 itens); Conforto físico-espacial, Orientação e Tranquilidade do serviço em geral (18 itens); Conforto físico-espacial e Vista e iluminação da área de internamento ou área de espera do serviço de consulta (21 itens); e Relações sociais e organizacionais, Privacidade e serviços complementares que pertencem à escala relativa aos aspectos sócio-funcionais (18 itens). Posteriormente, esse instrumento sofreu algumas alterações pelo facto de existir sobreposição entre as escalas de aspectos físico-espaciais do serviço em geral e da área de internamento/área de espera do serviço de consulta. Para contornar este problema, os autores juntaram essas escalas num instrumento que avalia quatro dimensões: Conforto físico-espacial, Orientação, Tranquilidade e Vista e iluminação (36 itens). As três escalas do PHEQI somavam um total de 72 itens, o que pode ser considerado repetitivo e cansativo, desencorajando a participação de doentes e profissionais de saúde, além de limitar a possibilidade de se adicionarem outras medidas ao protocolo de investigação.

Mais recentemente, a estrutura das escalas do PHEQI foi replicada noutro contexto cultural por Andrade, Lima, Fornara e Bonaiuto (2012), novamente em serviços de Ortopedia. Esses autores adaptaram e validaram o PHEQI através de uma amostra proveniente de quatro hospitais públicos portugueses. Os resultados de uma análise fatorial confirmatória atestaram a validade fatorial das escalas e obtiveram-se resultados bons ou satisfatórios em termos de consistência interna, validade de critério e validade de construto. Além disso, as escalas foram significativamente reduzidas em termos de número de itens.

No entanto, algumas questões ficaram por responder. Por um lado, as escalas do PHEQI necessitam de ser validadas noutros serviços hospitalares que não serviços de Ortopedia. Por outro lado, na validação para a população portuguesa, a dimensão Conforto físico-espacial da escala "Aspectos físico-espaciais do serviço em geral e área de internamento/área de espera do serviço de consulta" foi reduzida de 19 para seis itens, tendo sido eliminados, por exemplo, todos os itens que avaliavam questões relacionadas com a temperatura e qualidade do ar. Neste sentido, e de acordo com as recomendações fornecidas por Andrade et al. (2012) sobre a necessidade de continuar a estudar o PHEQI, pretendemos: a) testar se a ampla dimensão conceptual Conforto físico-espacial encontrada por Fornara et al. (2006) é melhor avaliada por duas dimensões em separado, sendo elas: Conforto físico-espacial e Temperatura e Qualidade dor
Ar; e b) testar a aplicabilidade das escalas do PHEQI noutros serviços hospitalares, nomeadamente em Unidades de Dor.

\section{Medir as Percepções da Qualidade do Ambiente em Unidades de Dor}

Tanto quanto sabemos, até o momento, nenhum estudo parece ter analisado diretamente as percepções que os doentes e profissionais de Unidades de Dor têm acerca dos aspectos ambientais do hospital/serviço. No entanto, Morais e Bernardes (2012) realizaram um estudo qualitativo, cujo objetivo consistiu na análise dos aspectos do ambiente físico e sócio-funcional que devem caracterizar uma Unidade de Dor ideal. Na opinião de doentes e profissionais de saúde, uma Unidade de Dor ideal deve ter em conta o conforto e a estética, proporcionando mobília de qualidade e elementos estético-decorativos. Além disso, aspectos relacionados com a temperatura e a qualidade do ar foram mencionados como importantes para um ambiente hospitalar de qualidade. Esses resultados indicam que a temperatura e a qualidade do ar são aspectos ambientais que deverão ser incluídos num instrumento cujo objetivo é medir a qualidade do ambiente hospitalar.

Assim, o presente estudo teve como objetivo validar as duas principais escalas do PHEQI em Unidades de Dor Portuguesas, testando a adequabilidade da (re)introdução de itens de medida da qualidade da temperatura e qualidade do ar na dimensão Conforto físico-espacial pertencente à escala de "Aspectos físico-espaciais do serviço em geral e área de internamento/área de espera do serviço de consulta". Para tal, foram reintroduzidos os itens da dimensão original (Fornara et al., 2006), que focam esses aspectos ambientais e que haviam sido removidos pela validação da escala para a população portuguesa (Andrade et al., 2012) (e.g., "A temperatura é inadequada - está demasiado quente ou demasiado frio") e adicionado um novo item ("O sistema de climatização do ar é eficiente"). A Tabela 1 apresenta a versão conceitual do instrumento alvo de estudo no presente trabalho.

\section{Método}

\section{Participantes}

Participaram neste estudo 122 utilizadores (86,7\% mulheres) de duas Unidades de Dor de Hospitais Públicos portugueses da zona da Grande Lisboa. A maioria dos participantes $(n=103)$ eram doentes e 19 eram profissionais de saúde. Os doentes (85,3\% mulheres) possuíam idades compreendidas entre os 34 e 84 anos $(M=64,0 ; \mathrm{DP}=12,0)$. A maioria (61,0\%) tinha apenas quatro anos de escolaridade e $68,7 \%$ estavam reformados ou reformados por invalidez. Noventa e oito por cento destes doentes já tinha visitado a Unidade de Dor pelo menos uma vez. Relativamente aos profissionais de saúde, estes possuíam idades compreendidas entre os 32 e os 59 anos $(M=46,0 ; D P=8,0)$ e trabalhavam na Unidade de Dor, em média, há sete anos $(D P=4,9)$. 
Tabela 1. Itens das Escalas do PHEQI

\begin{tabular}{l} 
Escala 1. Aspectos físico-espaciais do serviço em geral e áreas de \\
internamento e de espera do serviço de consulta \\
\hline Conforto físico-espacial (associado à mobília, paredes e tetos, \\
temperatura, etc.) \\
A mobília (armários, cadeiras, mesas, etc.) é de boa qualidade \\
As paredes, os pavimentos e os tectos têm cores bonitas \\
Os lugares sentados (ex: cadeiras, sofás) são pouco cómodos \\
As paredes, os pavimentos e os tectos estão em más condições \\
A mobília (camas, armários, cómodas, cadeiras, mesas, etc.) está em \\
boas condições \\
A mobília (camas, armários, cómodas, cadeiras, mesas, etc.) está em \\
más condições
\end{tabular}

Temperatura e qualidade do ar (temperatura, climatização e arejamento)

O nível de qualidade do ar é adequado (nem demasiado húmido, nem demasiado seco) ${ }^{1}$

A temperatura é inadequada (está demasiado quente ou demasiado frio $)^{1}$

O sistema de climatização do ar é eficiente ${ }^{2}$

$\mathrm{O}$ ar é irrespirável ${ }^{1}$

Orientação (características da áreas de internamento e de espera do serviço de consulta que facilitam a circulação e a orientação)

A entrada deste/a serviço/sala de espera é claramente reconhecível A sinalética permite encontrar facilmente aquilo que se procura Os locais onde se pedem informações estão claramente reconhecíveis Há poucos sinais para orientação

Vista e iluminação (presença de luz e qualidade da vista através da janela)

O/A serviço/sala de espera é pouco iluminado/a pela luz do sol

Das janelas tem-se uma vista pouco interessante

Devia haver mais janelas

Tranquilidade (nível de ruído)

Este serviço é barulhento ${ }^{2}$

Ouve-se frequentemente barulho proveniente do exterior

Ouvem-se poucos ruídos do exterior

Escala 2. Aspectos Sócio-funcionais

Relações sociais e organizacionais (relações com os profissionais de saúde e organização do serviço)

Neste serviço as pessoas recebem um bom acolhimento por parte dos profissionais de saúde

Em geral, o pessoal de enfermagem está pouco disponível do ponto de vista humano

Em geral, o pessoal médico está pouco disponível do ponto de vista humano

Este serviço é pouco organizado

Neste serviço há regras demasiado rígidas que limitam as pessoas

Em geral, o pessoal auxiliar está pouco disponível do ponto de vista humano

Privacidade (possibilidade de controlar a sua exposição e as interações com os outros)

As salas deste serviço estão frequentemente apinhadas de gente

Neste serviço tem-se a impressão de se estar a ser observado(a)

As pessoas criam muitos mexericos

Notas:

${ }^{1}$ Itens removidos durante a Análise Fatorial Confirmatória no estudo de

Andrade et al. (2012), relativo à adaptação e validação portuguesa do

PHEQI

${ }^{2}$ Novo item acrescentado na presente versão das escalas do PHEQI

\section{Instrumento}

Os participantes responderam a um protocolo que incluiu as duas escalas do PHEQI apresentadas na Tabela 1. As respostas aos itens das escalas são dadas numa escala de tipo Likert de cinco pontos de 0 "discordo totalmente" até 4 "concordo totalmente" e cada escala contém itens positivos (indicando a presença de qualidade) e negativos (indicando a ausência de qualidade), de forma a controlar a tendência à aquiescência nas respostas. Foi acrescentado um item à subescala Tranquilidade, uma vez que esta continha apenas itens acerca do barulho proveniente do exterior, nomeadamente: "Este serviço é barulhento". O item "A mobília (camas, armários, cómodas, cadeiras, mesas, etc.) está em más condições" da subescala Conforto físico-espacial foi retirado porque desta fazia também parte o item "A mobília (camas, armários, cómodas, cadeiras, mesas, etc.) está em boas condições", exatamente igual, mas pela positiva. Assim, a escala perfez um total de 19 itens.

Aos profissionais de saúde foi solicitado que avaliassem os "Aspectos físico-espaciais do serviço em geral" e aos doentes foi pedido que avaliassem, em separado, os aspectos físico-espaciais do serviço em geral e da sala de espera em particular. Desta forma, pudemos investigar como é que a escala se comporta quando é utilizada para avaliar as duas áreas em separado. Todos os participantes, doentes e profissionais de saúde, responderam à escala "Aspectos sócio-funcionais". Foi ainda recolhida informação acerca de características sociodemográficas tais como sexo, idade, grau de escolaridade e profissão.

\section{Procedimento}

Obteve-se o consentimento das Direções Hospitalares e das respectivas Unidades de Dor para a recolha de dados que foi realizada entre janeiro e maio de 2011 por uma investigadora treinada. Os doentes foram contactados maioritariamente na sala de espera após a consulta. Os profissionais de saúde foram abordados durante o seu horário de trabalho. A cada pessoa foi-lhes requerida a participação voluntária e referido que as suas respostas seriam confidenciais. Aos doentes que tinham dificuldades na leitura ou no preenchimento do questionário, este foi lido pela investigadora.

\section{Análise de dados}

Analisamos os dados por meio de um conjunto de Análises Factoriais Confirmatórias (AFC). Em todas as análises, os parâmetros foram estimados com base na matriz de variância-covariâncias dos itens da escala obtida após a eliminação pairwise dos dados omissos. Estimamos os parâmetros usando o método da máxima verosimilhança. Para assegurarmos a identificação estatística dos modelos, as variâncias dos fatores foram fixadas em 1,00. Consideramos adequados os modelos que apresentam valores de CFI (Comparative-Fit-Index) e GFI (Goodness-of-Fit-Index) superiores a 0,90, simultaneamente com RMSEA (RootMean-Square-Error-of-Application) inferiores a 0,08. De maior importância, comparamos a qualidade do ajustamento aos dados dos modelos propostos com o ajustamento de modelos alternativos. Essa análise foi realizada por meio da comparação dos valores de $\chi^{2}$ e de AIC (Akaike-InformationCriterion). 


\section{Resultados}

\section{Percepção da Qualidade do Ambiente Físico}

Uma inspeção inicial da matriz de correlações dos indicadores sugere que a maioria das correlações tem magnitude moderada e é significativa, o que sugere a possibilidade de existirem fatores subjacentes às relações entre as variáveis. De acordo com o modelo de medida proposto por Andrade et al. (2012), a percepção da qualidade do ambiente físico é constituída por quatro fatores correlacionados ${ }^{1}$. Uma vez que adicionamos dois itens para avaliar a temperatura e a qualidade do ar, hipotetizamos que o modelo apresente bom ajustamento se esses itens forem isolados num fator independente porque foram elaborados com base na hipótese de que há um quinto fator a orientar a percepção do ambiente físico, o qual chamamos "temperatura e qualidade do ar". Para testarmos a validade de uma estrutura fatorial composta por cinco fatores de primeira ordem, realizamos um conjunto de análises fatoriais confirmatórias. Os fatores foram especificados como variáveis latentes, representando cinco dimensões da percepção das pessoas sobre a qualidade do ambiente físico hospitalar: Conforto físico-espacial; Orientação; Tranquilidade; Vista e iluminação; Temperatura e qualidade do ar. As correlações entre os fatores foram especificadas para serem estimadas livremente.

\section{Percepção da qualidade do ambiente físico da sala de espera.}

A Figura 1 apresenta os parâmetros estimados para o modelo factorial hipotetizado aplicado à sala de espera.

Como podemos verificar, os itens apresentam pesos factoriais (loadings) elevados e significativos no fator para o qual foram especificados e existem correlações significativas entre os fatores, excepto entre os fatores Conforto físicoespacial e Tranquilidade. Particularmente relevante é uma correlação substancial entre os fatores Conforto físicoespacial e Temperatura e qualidade do ar.

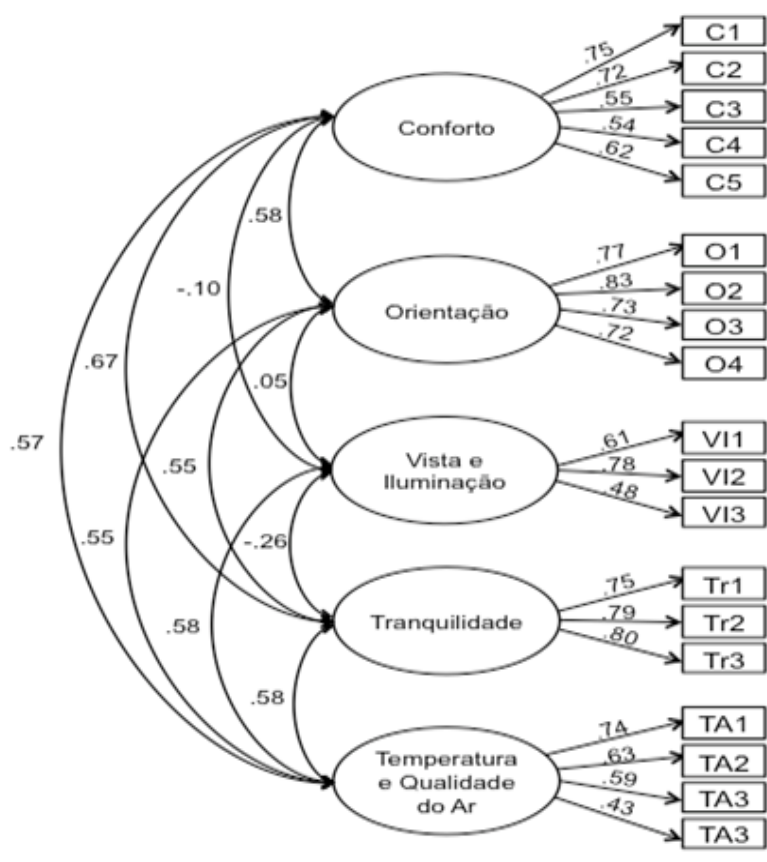

Figura 1. Análise Factorial Confirmatória da Escala de percepção da qualidade do ambiente físico da Sala de espera

A existência de uma correlação substancial entre os fatores sugere a presença de uma estrutura composta por cinco dimensões. Para avaliarmos esta possibilidade, comparamos o índice de ajustamento (goodness of fit) do modelo fatorial hipotetizado (Modelo 1) com o ajustamento de quatro estruturas fatoriais alternativas (Modelos 2, 3, 4 e 5 da Tabela 2).

No Modelo 2 especificamos uma estrutura fatorial constituída por apenas quatro fatores em que os itens das dimensões Conforto físico-espacial e Temperatura e qualidade do ar formaram um único fator. No Modelo 3 especificamos uma estrutura fatorial constituída por apenas um único fator de primeira ordem. Neste modelo, a hipótese é a de que todos os itens da escala medem apenas uma variável

Tabela 2. Goodness of fit das Diferentes Estruturas Factoriais da Escala de Medida da Percepção da Qualidade do Ambiente Físico da Sala de Espera

\begin{tabular}{|c|c|c|c|c|c|c|c|c|}
\hline & $\chi^{2}$ & gl & CFI & GFI & RMSEA & AIC & $\square \chi^{2}$ & $\Delta$ gl \\
\hline Modelo 1: Cinco factores correlacionados & $234,48 * * *$ & 147 & .87 & .81 & .08 & 320,48 & & \\
\hline Modelo 2: Quatro factores correlacionados & $322,28 * * *$ & 150 & .74 & .74 & .11 & 402,28 & & \\
\hline Modelo 2 vs. Modelo 1 & & & & & & & $87,8 * * *$ & 3 \\
\hline Modelo 3: Único Factor & $392,75 * * *$ & 152 & .63 & .70 & .13 & 468,78 & & \\
\hline Modelo 3 vs. Modelo 1 & & & & & & & $158,28 * * *$ & 5 \\
\hline Modelo 4: Cinco factores não correlacionados & $31913 * * *$ & 157 & .75 & .73 & .10 & 385.13 & & \\
\hline Modelo 4 vs. Modelo 1 & & & & & & & $84.66^{* * *}$ & 10 \\
\hline Modelo 5: Um factor geral de segunda ordem & $195.27 * * *$ & 147 & .93 & .83 & .06 & 281.27 & & \\
\hline
\end{tabular}

Nota. $C F I=$ Comparative Fit Index; GFI=Goodness of Fit Index; RMSEA=Root-Mean-Squared Error of Approximation. AIC=Akaike Information Criteria; $g l=$ Graus de Liberdade. $* * * p<.001$

1 As matrizes de correlações usadas nas análises apresentadas neste artigo estão à disposição dos leitores que as solicitarem aos autores por e-mail. 
latente (i.e., uma percepção global e indiferenciadas da qualidade do ambiente).

O Modelo 4 especifica uma estrutura fatorial idêntica ao Modelo 1 em número de fatores, mas assume a hipótese de que os cinco fatores são completamente independentes, i.e., não são correlacionados. No Modelo 5, especificamos uma estrutura fatorial formada por seis fatores, contendo os cinco fatores de primeira ordem hipotetizados no Modelo 1, e um fator geral de segunda ordem. A hipótese testada é a de que as cinco dimensões representadas nos fatores de primeira ordem são diferentes dimensões de um mesmo fator latente: percepção da qualidade do ambiente físico.

Os resultados mostram que o modelo hipotetizado (Modelo 1) apresenta índices de ajustamento razoáveis e que estes índices estão entre os melhores dos modelos testados (ver a coluna dos $\Delta \chi^{2}$ na Tabela 2). De facto, o Modelo 1 ajusta-se significativamente melhor aos dados do que os Modelos 2, 3 e 4. No entanto, o Modelo 1 não tem um ajustamento significativamente melhor do que o Modelo 5 , em que os cinco fatores de primeira ordem saturam num fator geral de segunda ordem. Isto sugere que o Modelo 5 é o que melhor descreve a percepção dos inquiridos sobre o ambiente físico na medida em que é mais parcimonioso (i.e., tem mais graus de liberdade) do que o Modelo 1, além de apresentar menor valor de AIC.

Assim, o conjunto dos resultados nos leva a aceitar uma estrutura fatorial com os cinco factores de primeira ordem a saturarem num fator geral de segunda ordem como a mais adequada para explicar as correlações entre os itens da escala de qualidade do ambiente físico.

\section{Percepção da qualidade do ambiente físico do serviço em geral.}

A Figura 2 apresenta os parâmetros estimados para o modelo fatorial hipotetizado aplicado ao serviço em geral. Os itens apresentam pesos fatoriais (loadings) elevados e significativos no fator para o qual foram especificados e existem correlações significativas entre os

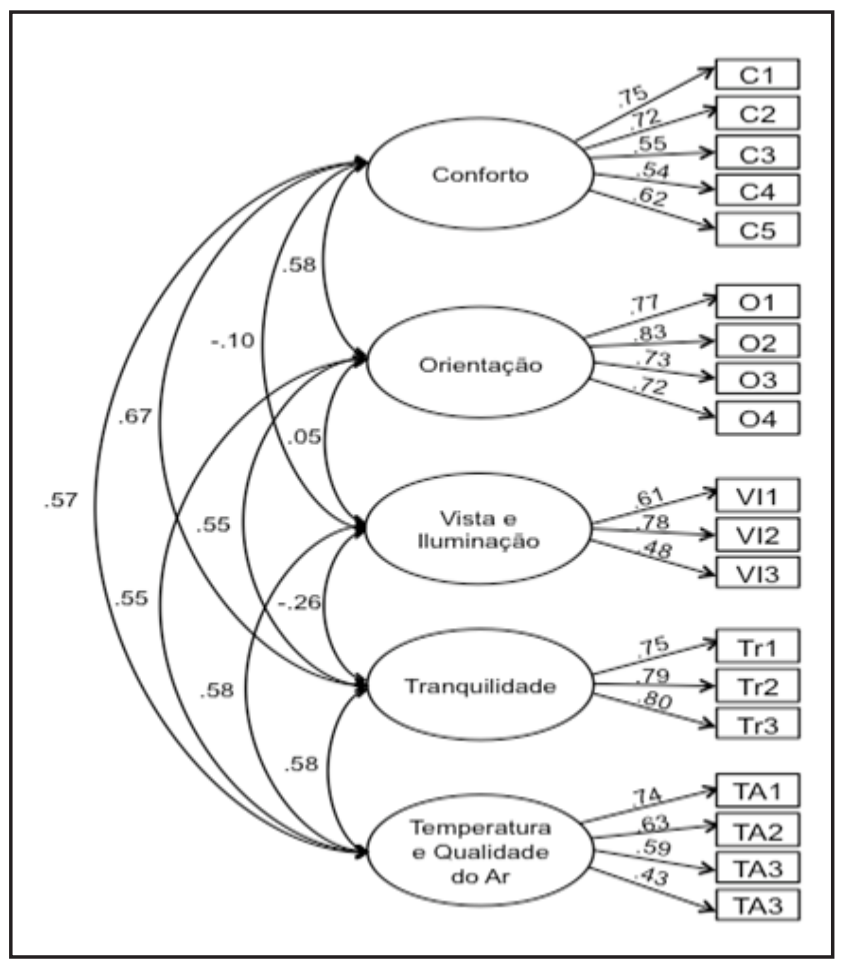

Figura 2. Análise Factorial Confirmatória da Escala de medida da percepção da qualidade do ambiente físico do Serviço em Geral

fatores, nomeadamente entre Conforto físico-espacial e Tranquilidade. As únicas correlações não significativas entre fatores são entre Vista e iluminação e Conforto físico-espacial e entre Vista e iluminação e Orientação. Por outro lado, Vista e iluminação correlaciona-se de forma significativa mas negativa com os fatores Tranquilidade e Temperatura e qualidade do ar. Esses resultados apontam que um modelo de cinco fatores não correlacionados (Modelo 4) poderia ter um ajustamento adequado. Tal como fizemos com o modelo aplicado à sala de espera, comparamos o índice de ajustamento do Modelo 1 com o ajustamento de quatro estruturas fatoriais alternativas (Modelos 2, 3, 4 e 5 da Tabela 3). Os resultados mostram que o modelo hipotetizado

Tabela 3. Goodness of fit das Diferentes Estruturas Factoriais da Escala de Medida da Percepção da Qualidade do Ambiente Físico do Serviço em Geral

\begin{tabular}{|c|c|c|c|c|c|c|c|c|}
\hline & $\chi^{2}$ & gl & CFI & GFI & RMSEA & AIC & $\square \chi^{2}$ & $\Delta \mathbf{g l}$ \\
\hline Modelo 1: Cinco factores correlacionados & $218.00 * * *$ & 142 & .90 & .85 & .67 & 314.00 & & \\
\hline Modelo 2: Único Factor & $471.40 * * *$ & 152 & .58 & .70 & .13 & 547.40 & & \\
\hline Modelo 2 vs. Modelo 1 & & & & & & & $243.51 * * *$ & 5 \\
\hline Modelo 3: Cinco factores não correlacionados & $344.23 * * *$ & 157 & .75 & .78 & .99 & 410.23 & & \\
\hline Modelo 3 vs. Modelo 1 & & & & & & & $116.34 * * *$ & 10 \\
\hline Modelo 4: Quatro factores & $286.23 * * *$ & 150 & .82 & .81 & .87 & 366.23 & & \\
\hline Modelo 4 vs. Modelo 1 & & & & & & & $58.34 * * *$ & 3 \\
\hline Modelo 5: Um factor geral de segunda ordem & $226.91 * * *$ & 147 & .90 & .85 & .67 & 312.91 & & \\
\hline Modelo 5 vs. Modelo 1 & & & & & & & 8.91 & 5 \\
\hline Modelo 5a: & $229.11 * * *$ & 149 & .90 & .85 & .06 & 313.11 & & \\
\hline Modelo 5a vs. Modelo 5 & & & & & & & 2.20 & 1 \\
\hline
\end{tabular}

Nota. $C F I=$ Comparative Fit Index; GFI=Goodness of Fit Index; RMSEA=Root-Mean-Squared Error of Approximation. AIC=Akaike Information Criteria; $g l=$ Graus de Liberdade. $* * * p<.001$ 
(Modelo 1) apresenta índices de ajustamento razoáveis comparados com os índices de ajustamento dos restantes modelos testados (ver a coluna dos $\Delta \chi^{2}$ Tabela 3 ). De facto, o Modelo 1 ajusta-se significativamente melhor aos dados do que os Modelos 2, 3 e 4. A comparação entre o Modelo 1 e o Modelo 5 indica haver diferença não significativa entre eles. No entanto, o Modelo 5 é mais parcimonioso e portanto descreve melhor a percepção dos inquiridos do que o Modelo 1, além de ser o modelo com menor valor de AIC. Assim, similar ao que verificamos sobre a percepção da qualidade do ambiente da sala de espera, os resultados indicam que uma estrutura fatorial com os cinco fatores de primeira ordem a saturarem num fator geral de segunda ordem é a melhor para explicar as correlações entre os itens da escala de percepção da qualidade do ambiente físico do serviço em geral. No entanto, ao inspecionarmos os pesos factoriais (loadings) dos fatores de primeira ordem no fator de segunda ordem designado "percepção geral da qualidade do ambiente físico do serviço em geral", constatamos que o peso do fator Vista e iluminação não é significativo. Isso indica que esse fator não é uma componente da percepção geral da qualidade do ambiente como o são os outros fatores. Nesse caso, realizamos uma análise suplementar na qual reespecificamos o Modelo 5 em que constrangemos a zero o peso do fator Vista e iluminação (Modelo 5a). Os resultados mostram um ajustamento adequado aos dados, o qual não difere significativamente do ajustamento do Modelo 5, embora seja mais parcimonioso. Esse resultado indica que, de facto, a percepção sobre a vista e iluminação não é uma componente da avaliação geral sobre a qualidade do ambiente físico dos serviços.

\section{Percepção da Qualidade do Ambiente Sócio-funcional}

A inspeção da matriz de correlações dos indicadores usados na avaliação da percepção da qualidade do ambiente sócio-funcional hospitalar mostrou a presença de correlações com magnitude moderada e significativa, o que sugere a possibilidade de existirem fatores subjacentes às relações entre as variáveis. A escala de percepção do ambiente sóciofuncional contém apenas oito itens elaborados para medir dois fatores, designados Relações sociais e organizacionais e Privacidade. A Figura 3 apresenta os parâmetros estimados para o modelo fatorial hipotetizado. Como se pode verificar, os itens apresentam pesos fatoriais (loadings) elevados e significativos no fator para o qual foram especificados, exceto o item 1, cujo peso fatorial, embora significativo, é inferior a 0,30, o que nos sugere eliminá-lo da escala. Também como previsto, a correlação entre os fatores Relações sociais e organizacionais e Privacidade é positiva e significativa.

Comparamos o índice de ajustamento do modelo fatorial hipotetizado (Modelo 1) com o ajustamento de três estruturas fatoriais alternativas (Modelos 2, 3 e 4 da Tabela 4). No Modelo 2 especificamos uma estrutura fatorial constituída apenas por um único fator de primeira ordem. Nesse modelo, a hipótese é a de que todos os itens da escala medem apenas uma variável latente. O Modelo 3 especifica uma estrutura fatorial idêntica ao Modelo 1 em número de fatores, mas assume a hipótese de que os dois fatores são completamente independentes, i.e., não são correlacionados. No Modelo 4 especificou-se uma estrutura fatorial formada por dois fatores de primeira ordem hipotetizados no Modelo 1 e um fator geral de segunda ordem para testar se os dois fatores de primeira ordem são diferentes dimensões de um mesmo fator latente: percepção da qualidade do ambiente sócio-funcional. Os resultados mostram que o modelo hipotetizado (Modelo 1) apresenta índices de ajustamento razoáveis e melhores do que os índices de ajustamento dos modelos alternativos.

Realizamos análises suplementares e com o objetivo de melhorar o ajustamento do modelo retiramos o item 1 do factor Relações sociais e organizacionais. De facto, quando retiramos o item 1 (Modelo 1a), o ajustamento do modelo aos dados passa a ser muito bom. Observamos também os índices de modificação post hoc para o modelo que nos sugeriram a ocorrência de correlações significativas entre os erros de medida das seguintes variáveis: E2 e E3 do fator "relações sociais e organizacionais" $(r=.57, p<.001)$ e E6 e E7 do fator "privacidade" ( $r=.41, p<.001)$. Reespecificamos o modelo permitindo que essas correlações fossem estimadas

Tabela 4. Goodness of fit das Diferentes Estruturas Factoriais da Escala de Medida da Percepção da Qualidade do Ambiente SócioFuncional

\begin{tabular}{|c|c|c|c|c|c|c|c|c|}
\hline & $\chi^{2}$ & gl & CFI & GFI & RMSEA & AIC & $\square \chi^{2}$ & $\Delta \mathbf{g l}$ \\
\hline Modelo 1: Dois factores correlacionados & $66.67 * * *$ & 26 & .83 & .89 & .12 & 104.67 & & \\
\hline Modelo 2:Um único Factor & $103.11 * * *$ & 27 & .67 & .83 & .16 & 139.11 & & \\
\hline Modelo 2 vs. Modelo 1 & & & & & & & $36.44 * * *$ & 1 \\
\hline Modelo 3: Dois factores não correlacionados & $72.03 * * *$ & 27 & .81 & .88 & .12 & 108.03 & & \\
\hline Modelo 3 vs. Modelo 1 & & & & & & & $5.36^{*}$ & 1 \\
\hline Modelo 4: Um factor de $2^{\mathrm{a}}$ ordem & $103.11 * * *$ & 27 & .67 & .83 & .16 & 139.11 & & \\
\hline Modelo 4 vs. Modelo 1 & & & & & & & $36.44 * * *$ & 1 \\
\hline Modelo 1a: Dois factores correlacionados Sem item 1 & $59.85^{* * *}$ & 19 & .82 & .88 & .14 & 93.85 & 6.82 & \\
\hline $\begin{array}{l}\text { Modelo 1b: Dois factores correlacionados Sem item } 1 \\
\text { Correlações e16-e17; e9-e8 }\end{array}$ & 22.70 & 17 & .98 & .95 & .05 & 60.70 & & \\
\hline Modelo 1b vs. Modelo 1a & & & & & & & $37.15^{* * *}$ & 2 \\
\hline
\end{tabular}

Nota. $C F I=$ Comparative Fit Index; GFI=Goodness of Fit Index; RMSEA=Root-Mean-Squared Error of Approximation. AIC=Akaike Information Criteria; $g l=$ Graus de Liberdade. $* * * p<.001$ 
livremente (Modelo 1b). Os resultados nos permitiram verificar um ajustamento muito bom desse modelo aos dados, de modo a nos permitir aceitar que escala mede uma estrutura fatorial composta por os dois fatores de primeira ordem correlacionados, sem o item 1 e com duas correlações entre erros. Essa estrutura é a que melhor explica as correlações entre os itens da escala de qualidade do ambiente sóciofuncional.

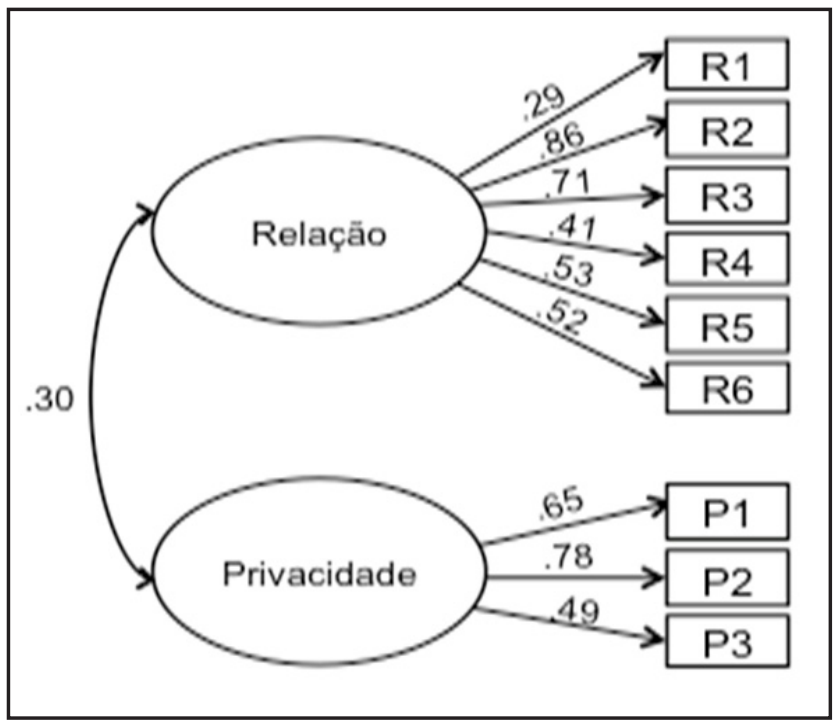

Figura 3. Análise Factorial Confirmatória da Escala de medida da percepção da qualidade do Ambiente sócio-funcional

\section{Discussão dos Resultados}

Este estudo teve como objetivo testar a validade de duas escalas de medida da percepção da qualidade do ambiente hospitalar em Unidades de Dor: uma para medir a qualidade do ambiente físico e outra para medir a qualidade do ambiente sócio-funcional. O segundo objetivo foi aprofundar o estudo da escala de medida da percepção da qualidade do ambiente físico em termos das suas dimensões conceituais. Em particular, testamos um modelo de medida composto não por quatro (Orientação, Tranquilidade, Vista e iluminação e Conforto físico-espacial)), mas por cinco dimensões, incluindo a dimensão Temperatura e qualidade do ar.

A melhor solução fatorial para a percepção da qualidade do ambiente físico da sala de espera é a proposta pelo modelo que organiza os cinco fatores (Orientação, Tranquilidade, Vista e iluminação e Conforto físico-espacial e Temperatura e qualidade do ar) num fator geral de segunda ordem. Este resultado confirma a hipótese de que a dimensão Temperatura e qualidade do ar faz sentido ser considerada como descrevendo a qualidade percebida desse ambiente (Morais \& Bernardes, 2012). Em relação à percepção da qualidade do ambiente físico do serviço em geral, a melhor solução fatorial foi também a proposta pelo modelo com um fator geral de segunda ordem. No entanto, a componente Vista e iluminação não explicou a qualidade percebida do ambiente físico do serviço em geral, sendo melhor representada como um fator independente. Parece-nos que este resultado pode ser explicado pela forma como os utilizadores percepcionam as duas áreas hospitalares avaliadas: a sala de espera e o serviço em geral. A sala de espera diz respeito à área do serviço onde os doentes aguardam pela consulta, frequentemente durante longos períodos de tempo. Nessa área, a vista e iluminação são critérios importantes: a vista como fator distrator e que ajuda a regular a percepção do tempo, e a iluminação que permite que as pessoas possam ler ou realizar outras atividades enquanto esperam pela consulta. Por outro lado, o serviço em geral refere-se a toda a área que circunda a sala de espera, nomeadamente, a entrada do serviço e os corredores que ligam as várias zonas. De facto, uma vez que esses espaços são maioritariamente interiores e de passagem, ou onde os doentes e profissionais passam pouco tempo, é plausível que a avaliação da qualidade do ambiente físico seja feita com base em critérios relacionados com a orientação, a tranquilidade, o conforto e a temperatura e qualidade do ar, mas não com base na vista e iluminação.

Em relação à qualidade do ambiente sócio-funcional, o modelo que melhor se adequou aos dados é aquele que representa os fatores Privacidade e Relações sociais e organizacionais como fatores de primeira ordem correlacionados. Vale a pena notar que a escala original publicada por Fornara et al. (2006) continha itens relacionados com informação sobre os exames e os procedimentos médicos, satisfação com a visita do médico e clima de colaboração entre os profissionais, entre outros, que foram eliminados na adaptação da escala à população portuguesa. Neste estudo, 0 item "Neste serviço as pessoas recebem um bom acolhimento por parte dos profissionais de saúde" foi também eliminado para melhorar o ajustamento do modelo. $\mathrm{O}$ facto de o padrão de respostas a este item ser enviesado positivamente, com a maioria dos inquiridos a responder "concordo totalmente" $(\mathrm{M}=3.87, \mathrm{DP}=.66)$, indica que poderá ser vulnerável ao efeito da desejabilidade social. Assim, é possível que essa escala esteja a negligenciar outras componentes importantes da percepção da qualidade dos cuidados. Sugerimos que estudos futuros desenvolvam uma subescala que inclua aspectos como a informação sobre o diagnóstico, procedimentos e prognóstico, a comunicação entre os profissionais de saúde e os doentes, a participação do doente - que a literatura tem mostrado serem muito importantes para a satisfação dos doentes e adesão terapêutica (Joffe et al., 2003) e que poderão definir a qualidade dos aspectos sócio-funcionais do serviço hospital. Também a subescala Privacidade parece não possuir elevada validade facial, por exemplo, não inclui aspectos relacionados com a privacidade auditiva, isto é, poder falar-se de assuntos privados sem se ser ouvido, que naturalmente são importantes tanto nas consultas médicas como em serviços de internamento (Lin \& Lin, 2011). Concluindo, sugerimos que, tal como a escala de medida da qualidade do ambiente físico contém várias componentes, também a escala de medida da qualidade do ambiente sócio-funcional poderá se beneficiar se contiver mais componentes, mais específicas em termos de conteúdo, e mais completas.

$\mathrm{O}$ estudo aqui apresentado foi o primeiro a comparar vários modelos de medida. Os resultados encontrados sustentam a possibilidade de se poder usar uma medida global para a qualidade do ambiente físico da sala de espera. No entanto, o mesmo só se pode fazer para a qualidade do ambiente físico do serviço em geral se não se incluir a subescala Vista e iluminação, que deve ser analisada 
separadamente. Quanto às subescalas Privacidade e Relações sociais e organizacionais da escala de medida da qualidade do ambiente sócio-funcional, também estas devem ser analisadas em separado.

Os resultados apresentados permitem confirmar que as escalas do PHEQI são medidas fiáveis e válidas para aceder à forma como os utilizadores do hospital avaliam a qualidade do ambiente físico e do ambiente sócio-funcional noutros serviços, como é o caso das Unidades de Dor. No entanto, mais estudos devem ser feitos com amostras diversificadas e em contextos culturais distintos de modo a que a sua utilização possa ser generalizada, bem como para testar as hipóteses aqui levantadas. Além disso, a adequação das escalas, com estas melhorias, deve ser também testada em serviços de internamento. Apesar dessas limitações, o instrumento aqui descrito é útil dentro da perspectiva dos cuidados de saúde centrados no doente e na melhoria da qualidade do ambiente de trabalho dos profissionais de saúde, em que o ambiente físico pode (deve) ser intencionalmente gerido como favorecedor e não constrangedor do bem-estar.

\section{Referências}

Andrade C. C., Lima, M. L., Fornara, F., \& Bonaiuto, M. (2012). What is a hospital with environmental quality? - Validation and further development of a hospital environmental quality perception measure. Journal of Environmental Psychology, $32,97-111$.

Andrade, C. C., Hernández-Fernaud, E., \& Lima, M. L. (2013). A better physical environment in the workplace means higher well-being? - A study with healthcare professionals. Psyecology, 4(1), 89-110.

Bell, P. A., Greene, T. C., Fisher, J. D., \& Baum, A. (2001). Environmental Psychology (5th ed.). Fort Worth: Harcourt College Publishers.
Bonaiuto, M., Fornara, F., \& Bonnes, M. (2003). Indexes of perceived residential environment quality and neighbourhood attachment in urban environments: A confirmation study on the city of Rome. Landscape and Urban Planning, 65(1-2), 41-52.

Bonnes, M., \& Secchiaroli, G. (1995). Environmental Psychology. A psycho-social introduction. London: Sage.

Chaudhury, H., Mahmood, A., \& Valente, M. (2009). The effect of environmental design on reducing nursing errors and increasing efficiency in acute care settings: A review and analysis of the literature. Environment and Behavior, 41(6), 755-786.

Devlin, A. S., \& Arneill, A. B. (2003). Health care environments and patient outcomes: A review of the literature. Environment \& Behavior, 35(5), 665-694.

Fornara, F., Bonaiuto, M., \& Bonnes, M. (2006). Perceived hospital environment quality indicators: A study of orthopaedic units. Journal of Environmental Psychology, 26, 321-334.

Joffe, S., Manocchia, M., Weeks, J. C., Cleary, P. D., Manocchia, N., \& Geary, P. D. (2003). What do patients value in their hospital care? An empirical perspective on autonomy centred bioethics. Journal of Medical Ethics, 29(2), 103-108.

Lin, Y. -K., \& Lin, C. -J. (2011). Factors predicting patients' perception of privacy and satisfaction for emergency care. Emergency Medicine Journal, 28(7), 604-608.

Moos, R. H., \& Lemke, S. (1984). Multiphasic environmental assessment procedure: Manual. Palo Alto, CA: Social Ecology Laboratory, Veterans Administration and Stanford University Medical Center.

Morais, R., \& Bernardes, S. (2012). Percepções sobre o ambiente físico e sócio-funcional de uma Unidade de Dor Portuguesa: Estudo qualitativo com utentes e profissionais de saúde. Dor, 20(3), 39-48.

Ulrich, R. (1984). View through a window may influence recovery from surgery. Science, 224(4647), 420-421.

Recebido em 25.05.2013

Primeira decisão editorial em 27.10.2014

Versão final em 12.12.2014

Aceito em 12.12.2014 